\title{
Awareness of thrombotic disease during lockdown: an unusual consequence of the COVID-19 pandemic
}

\author{
Matthaios Speletas $^{1}$ iD
}

Accepted: 27 November 2020 / Published online: 2 January 2021

(c) The Author(s), under exclusive licence to Springer Science+Business Media, LLC part of Springer Nature 2021

\section{Dear Sir,}

The coronovirus disease 2019 (COVID-19) pandemic is currently one of the foremost health challenges worldwide. Most countries have now re-implemented lockdown-focused policies in order to limit the transmission of severe acute respiratory syndrome coronavirus 2 (SARS-CoV-2) and restrict its spread. Although current interest has focused on the socio-economic consequences of lockdown due to COVID-19, complications to public health should not be diminished. I consider that an awareness of specific health problems should always been taken into consideration. In this article I exhibit a case study supporting my view.

Twenty-one days after the first strict national lockdown was enforced in Greece in March 2020, a non-obese and nonsmoking 18-year-old male student complained of pain in the lower left abdomen and swelling of the left femur which he experienced for the previous three days. The patient had only a medical history of tonsillectomy and adenoidectomy during childhood, while his father suffered from superficial thromboses on varicose veins of the lower extremities. Initial lab results, including a complete blood count, biochemistry and urinalysis, were normal; however, a computer tomography (CT) of the patient's abdomen and a doppler ultrasound of his legs revealed the presence of thrombus in the left iliac and common femoral veins. Antiphospholipid antibodies were within normal limits, the CT scan of the thorax and ultrasound of testicles were normal, while a comprehensive coagulopathy workup revealed only a factor V Leiden (FVL, F5-c.1691G > A) heterozygosity. It is noteworthy that the patient's parents emphasized that since the beginning of the national lockdown, their son spent over $10 \mathrm{~h}$ per day continuously seated in front of his computer.

Matthaios Speletas

maspel@med.uth.gr; speletas@gmail.com

1 Department of Immunology \& Histocompatibility, Faculty of Medicine, University of Thessaly, 41500 Larissa, Greece
Since the patient started receiving tinzaparin and attended an outpatient clinic, his condition gradually improved. Today, he remains in excellent condition under warfarin treatment.

The patient's medical condition, characterized by deep vein thrombosis (DVT) in the context of inherited thrombophilia and prolonged sitting due to the lockdown, may be considered a variant of "economy-class syndrome" (ECS). In ECS, patients presented DVT with or without pulmonary thromboembolism after a prolonged airplane flight in economy class seats, attributed to prolonged immobility in cramped conditions [1,2]. Additional studies reported that ECS can also be attributed to long automobile or train trips ("travelers' thrombosis"), or prolonged periods of being seated in an unconformable position in automobiles after an earthquake or in war shelters [3, 4]. Similar to my patient, the majority of them displayed predisposing risk factors [2].

It is evident that preventive measures, including avoidance of prolonged unconformable sitting, exercising at home, and high fluid intake should be recommended during the coronavirus lockdown. Obviously, such recommendations should be implemented for the entire society, bearing in mind that the liable risk factors-such as heritable theombophilia (as the described case), hypertension, obesity, and diabetes mellitus-are often underdiagnosed and/or underestimated, contributing to an increased morbidity and mortality during the restrictive measures. In this context, it is also important to recommend physical and/or mental breaks for all virtual activities, including scientific meetings and conferences. I consider that such recommendations should be included in any government's guidelines during the current COVID-19 lockdown or other similar emergencies, in order to prevent thrombotic attacks and their consequent complications.

Funding No funds, grants, or other support was received. 


\section{Compliance with ethical standards}

Conflict of interest There is no conflict of interest related to this article.

\section{References}

1. Cruickshank JM, Gorlin R, Jennett B (1998) Air travel and thrombotic episodes: the economy class syndrome. Lancet 2(8609):497498. https://doi.org/10.1016/s0140-6736(88)90134-1

2. Arya R, Barnes JA, Hossain U, Patel RK, Cohen AT (2002) Longhaul flights and deep vein thrombosis: a significant risk only when additional factors are also present. Br J Haematol 116:653-654. https://doi.org/10.1046/j.0007-1048.2001.03330.x

3. Yokota T, Kojima S, Yamauchi H (2006) Hatori M (2006) Regional medicine following a large earthquake: economyclass syndrome. Disaster Manag Response 4:3. https://doi. org/10.1016/j.dmr.2005.10.003

4. Dalen JE (2003) Economy class syndrome: too much flying or too much sitting? Arch Intern Med 163:2674-2676. https://doi. org/10.1001/archinte.163.22.2674

Publisher's Note Springer Nature remains neutral with regard to jurisdictional claims in published maps and institutional affiliations. 